Macromolecular Nanotechnology

\title{
Control of molecular weight and polydispersity in polyethylene/needle-like shaped clay nanocomposites obtained by in situ polymerization with metallocene catalysts
}

\author{
Manuel Herrero $^{\mathrm{a}, *}$, Karina Núñez ${ }^{\mathrm{b}}$, Raúl Gallego ${ }^{\mathrm{b}}$, Juan Carlos Merino ${ }^{\mathrm{a}, \mathrm{b}}$, José María Pastor ${ }^{\mathrm{a}, \mathrm{b}}$ \\ ${ }^{a}$ Departament of Condensed Matter Physics, Escuela de Ingenierías Industriales, University of Valladolid, Paseo del Cauce, 59,47011 Valladolid, Spain \\ ${ }^{\mathrm{b}}$ Foundation for Research and Development in Transport and Energy (CIDAUT), Parque Tecnológico de Boecillo, 47051 Valladolid, Spain
}

\section{A R T I C L E I N F O}

\section{Article history:}

Received 29 June 2015

Received in revised form 4 December 2015

Accepted 11 December 2015

Available online 12 December 2015

\section{Keywords:}

In situ polymerization

Metallocene catalyst

Polyethylene (PE)

Sepiolite nanoparticles

Mechanical and rheological balance

\begin{abstract}
A B S T R A C T
In an effort to understand the influence of the shape and area of the nanoclay type used in the in situ polymerization process, two different types of clays (fibrillar and laminar) were employed to obtain nanocomposites. The preliminary results demonstrated that a needlelike shaped clay promotes higher molar mass and better mechanical properties compared with laminar clay. The ultra-high molecular weight of sepiolite/nanocomposites introduces significant problems with processability. Therefore, a new method of polymerization was designed to include two significant changes. The first change was related to the use of a non-isothermal temperature profile and the second to the addition of an additional amount of non-anchored co-catalyst leading to materials with higher fluidity.
\end{abstract}

(c) 2015 Elsevier Ltd. All rights reserved.

\section{Introduction}

Although they were discovered more than a half century ago, polyolefins are materials that are still in high demand. Polyolefins are thermoplastics that are simple in structure and economical in terms of production and processing. In addition, the synthesis of polyolefins has evolved to adapt these materials to the needs of current consumption. Currently, approximately 60 million tonnes of polyolefins are produced each year, but the predominant uses are limited to commodity applications (i.e., packaging, manufacturing pipes, insulating materials and industrial parts). In other applications, such as in the automotive sector, there is less interest in this type of polymer because required mechanical parameters are out of reach, and blending polyolefins with other polymers or fillers is impossible due to the absence of polar groups on its structure.

Fortunately, creating nanocomposites extends the usefulness of polyolefins. These nanocomposites are hybrid materials consisting of an organic polymer matrix dispersed with an inorganic filler with least one dimension in the nanometre scale $[1,2]$. Adding a very small amount of nanofiller increases the stiffness of the matrix. However, the full potential of nanocomposites is only exploited when the nanofiller is homogeneously dispersed within the polymer matrix.

Polymer nanocomposites can be prepared via three routes, namely solution blending [3-5], melt blending [6-11] and in situ polymerization [12-14]. The most common commercially implemented procedure to obtain nanocomposites is melt blending. However, depending on the type of load, the experimental procedure and the matrix, materials with poor mechanical properties are sometimes obtained because of low interfacial tension between the components [15]. Furthermore, when

\footnotetext{
* Corresponding author.

E-mail address: manher@cidaut.es (M. Herrero).
} 
the fillers are particles with sizes less than $0.1 \mu \mathrm{m}$, achieving good dispersions in the polymer matrix is difficult via conventional melt processes due to the tendency of these nanoparticles to form clumps [16]. The most common way to improve the compatibility between the load and the matrix is to chemically modify the surface of the filler using compatibilizing agents [17].

The goal of in situ polymerization is the insertion of the nanofiller in the polymer matrix during the polymer synthesis process. This method enables making in a single step, thus increasing the compatibility and the dispersion of the nanofiller. The idea of fabricating nanocomposites via in situ polymerization has evolved simultaneously with investigations into the immobilization of metallocene catalysts onto nanoparticle surfaces [18-20].

In the year 2000, the number of scientific reports on in situ polymerization began to increase. In the first studies, different types of micro-sized fillers (i.e., kaolin, silica (acidic surfaces), glass spheres, magnesium hydroxide (basic surface), nickel and graphite) were integrated into the polymerization process. The majority of reports only managed to prove that the method was able to form a thin layer of polymer on the surface of the fillers. However, the productivity of the reaction was severely affected by the increase in hetero-atoms present in the reaction, which simultaneously caused a loss of catalytic activity. Therefore, the percentages of load in the synthesized materials were also very high (17-30 wt.\%) [21,22].

Between 2002 and 2004, when investigations on nanocomposite technology were the most frequent, montmorillonite, hectorite or bentonite were incorporated into in situ polymerizations were published $[23,24]$. The reactions were conducted with a metallocene catalyst supported by the nanoparticles. Although very productive reactions were reported, the ultrahigh molecular weight of the matrix became one of the most significant problems for this method. The authors concluded that increasing the clay content in the polymerization medium promoted an indiscriminate increase in molecular weight, leading to materials with serious problems achieving the proper fluidity for processing and moulding.

Later, in 2006-2008, authors began to exploit the use of other nanofillers (e.g., glass nanospheres, calcium carbonate, nanotubes and carbon nanofibres) for more specific applications [25,26]. Most of these fillers required a pre-treatment (e.g., calcinations to remove moisture or acid treatments) to promote the immobilization of catalyst on the load surface $[27,28]$. Nevertheless, independently of the type of nanofiller employed, the problem of increasing the molecular weight persisted. Additionally, polymers with high amounts of filler were obtained because the modifiers deactivated the catalytic system during the reaction.

Between the years 2008 and 2009 the first reports were published on controlling the molecular weight in in situ polymerization [29,30]. Most of these works concluded that transfer agents, such as hydrogen, should promote transference reactions and thus effectively control the molecular weight [31].

In addition to the difficulty of processing the nanocomposites synthesized via in situ polymerization, most of these studies reported a decrease or only a slight increase in the Young's modulus of these nanocomposites relative to the homologous homopolymers. In all cases, the structures of the synthesized polymers exhibited excellent dispersion and distribution of the nanofillers. Dubois et al. demonstrated that metallocene catalysts produce polyolefins with high and narrow molar mass distributions [21-23]. Moreover, they specified that clay does not have a nucleant effect on a very ordered matrix per se. Therefore, controlling the molecular weight is vital to exploit the benefits on the mechanical properties when nanocomposites are obtained by in situ polymerization.

Furthermore, the majority of results reported until 2009 on in situ polymerizations incorporating laminar nanofillers. In 2010, the incorporation of a fibrillar-shaped clay (palygorskite) into in situ polymerization was reported for the first time to explore the possible benefits that this clay offered to the polymerization [32]. These authors reported a significant increase in Young's modulus (20\%) with $5 \mathrm{wt}$.\% of the final filler as well as a decrease in over $25 \%$ in the crystallinity compared with those parameters in neat polyethylene (PE).

In light of these results, the aim of this paper is to explore the formation of polyethylene/sepiolite nanocomposite by in situ polymerization using a metallocene/MAO system. Two different strategies for preparing these nanocomposites were explored to control the molecular weight and improve the nucleant effect of clay on the PE matrix. The first was based on a non-isothermal process and the second on adding an additional co-catalyst not anchored onto the surface of the clay in the polymerization medium. In the first stages of this investigation, two different clay shapes were compared to demonstrate that fibrillar clays are the most appropriate for in situ polymerization.

\section{Experimental}

\subsection{Materials}

Manipulations of oxygen and moisture sensitive materials were carried out under a controlled atmosphere. The clays used for the preparation of the metallocene PE nanocomposites were a commercial sepiolite (SEP) supplied by TOLSA and bentonite (BET) by KAO. These fillers were dried under vacuum at $80{ }^{\circ} \mathrm{C}$ for $24 \mathrm{~h}$ before treatment. Bis(Ciclopentadienyl)-Zir conium(IV) dichloride, $\mathrm{Cp}_{2} \mathrm{ZrCl}_{2}$, which was used as catalyst, was purchased from Aldrich and used as received.

Methylaluminoxane (MAO, 7 wt.\% solution in toluene, AzkoNobel) was used as the co-catalyst. Toluene was freshly dried by distillation from sodium-benzofenone under a nitrogen atmosphere. Polymerization-purity-grade ethylene supplied by Air Liquide was used after passing through an oxy trap and molecular sieves to remove the oxygen and water. 


\subsection{Clay pre-treatment}

The desired amount of dry clay (SEP) was mixed with the co-catalyst in various ratios ( $\mathrm{ml}$ of MAO solution:g clay) in $100 \mathrm{ml}$ of dry toluene and stirred for $90 \mathrm{~min}$ at room temperature following the procedure explained in our previous work on optimizing the immobilization of MAO onto the sepiolite surface [33]. The resulting solid (SEP-MAO) was then washed three times with $30 \mathrm{ml}$ of fresh toluene. The SEP-MAO was dried inside a glove box and stored there until immediately before use. The same procedure was employed to modify the BET surface with the MAO. The quantity of MAO, anchored onto the clay, particles is reported in Table 1.

\subsection{Polymerization}

Ethylene polymerizations were carried out in a 11 stainless steel autoclave equipped with a mechanical stirrer under the conditions explained in W02013-167764A1 [34].

\subsection{Preparation of test specimens}

A Schwabenthan vacuum press and a homemade mould were used to compression mould sheets of the polymer composites $\left(1.5 \times 200 \times 200 \mathrm{~mm}^{3}\right)$. The polymer (dry powder samples) was stabilized with $0.5 \mathrm{wt}$.\% of a 1:1 mixture of Irganox 1010 and Irgafos 168 to prevent oxidative and thermal degradations. An internal mixer was employed to homogenize the polymer powder and stabilizers. The fusion was carried out at $190^{\circ} \mathrm{C}$, the frequency of the rotors was set to $60 \mathrm{rpm}$, and the polymers were melted and mixed for $5 \mathrm{~min}$.

The moulding cycle was as follows: the melt polymer was kept in the mould for $3 \mathrm{~min}$ without pressure and then compressed for $5 \mathrm{~min}$, both at $190^{\circ} \mathrm{C}$. The moulded sheets were cooled down to room temperature using an internal cold water circuit. The samples were cut accordingly to the requirements for each characterization test.

\subsection{Characterization}

X-Ray diffraction (XRD) was performed on the clays and nanocomposites in a Philips X'Pert MPD using Cu K $\alpha$ radiation source to evaluate the evolution of the clay's $\mathrm{d}_{001}$ reflection.

The melt and crystallization temperatures as well as the heats of fusion and crystallization of the samples were measured by differential scanning calorimetry (DSC) with a Mettler-Toledo DSC model 821e thermal analysis system in the temperature range from 25 to $200{ }^{\circ} \mathrm{C}$ at a heating rate of $20^{\circ} \mathrm{C} \mathrm{min}^{-1}$ under nitrogen flow. The samples were first heated to $200{ }^{\circ} \mathrm{C}$ for 2 min to eliminate their thermal history and subsequently cooled to $25^{\circ} \mathrm{C}$. The second endotherm was recorded by heating at $20{ }^{\circ} \mathrm{C} \mathrm{min}^{-1}$, and the degree of crystallinity, $T_{m}$ and $\Delta H_{m}$ of the samples were calculated. The heat of fusion of neat PE is $293 \mathrm{~J} \mathrm{~g}^{-1}$. The successive self nucleation and annealing (SSA) experiment was carried out by establishing self-nucleation isothermal steps for $5 \mathrm{~min}$ at each temperature and by decreasing the temperature by $5{ }^{\circ} \mathrm{C}$ after each step. Thermogravimetric analysis (TGA) was used to determine the clay content in the obtained nanocomposites. Thermograms were obtained in a nitrogen atmosphere at a heating rate of $10{ }^{\circ} \mathrm{C} \mathrm{min}^{-1}$ using a Mettler-Toledo TGA851.

To analyse the morphology of the nanocomposite, the surface of the obtained powder sample, was observed by scanning electron microscopy (SEM) in a Hitachi S-4700 Electron Microscope with an accelerating voltage of $20 \mathrm{kV}$, after coating with gold. Transmission electron microphotographs (TEM) were taken from $100 \mathrm{~nm}$ microtomed sections of the composites cut with a Reichert-Jung Ultracut E microtome using a JEOL JEM 2000FX Electron Microscope with an accelerating voltage of $200 \mathrm{kV}$.

The melt flow index was determined by measuring the polymer fluidity in the molten state at $190{ }^{\circ} \mathrm{C}$ and $2.16 \mathrm{Kg}$ according to ISO 1133. The density was determined by liquid immersion in accordance with the standard ISO 1183-1.

Molar mass distributions were determined with a Waters ALLIANCE GPCV 2000 gel permeation chromatographer (GPC) using 1,2,4-trichlorobenzene as the solvent and a flow rate of $1 \mathrm{~cm}^{3} \mathrm{~min}^{-1}$. The analyses were performed at $145^{\circ} \mathrm{C}$. A dynamical analysis (DMA) was carried out with a Mettler-Toledo at a fixed frequency $(1 \mathrm{~Hz})$ with a heating rate of $3 \mathrm{~K} \mathrm{~min}^{-1}$ between $-135^{\circ} \mathrm{C}$ and $145^{\circ} \mathrm{C}$.

Table 1

Amount of MAO anchored on the surface of clay with different treatments.

\begin{tabular}{ll}
\hline Sample $^{\mathrm{a}}$ & MAO on filler (wt.\%) \\
\hline SEP_1:2 & 1.1 \\
SEP_1:1 & 0.8 \\
SEP_2:1 & 0.6 \\
SEP_4:1 & 0.3 \\
BET_2:1 & 0.4 \\
\hline
\end{tabular}

\footnotetext{
${ }^{a}$ Nomenclature. SEP: sepiolite or BET: bentonite, $1: 2$ corresponding to [g
} initial clay:ml MAO solution]. 
The mechanical properties of the resulting nanocomposites were also tested. The Younǵs modulus and tensile strength were measured with an Instron Model 5500R60025 at a speed of $1 \mathrm{~mm} \mathrm{~min}^{-1}$ and $50 \mathrm{~mm} \mathrm{~min}^{-1}$ according to UNE-EN ISO 527-1 and 527-2, respectively.

Finally, the polyethylene chains were extracted from the nanocomposite using a Soxhlet extractor (decalin) for $24 \mathrm{~h}$ to evaluate the soluble fraction.

\section{Results and discussion}

\subsection{Influence of the clay shape}

Polyethylene nanocomposites were polymerized in the presence of MAO supported onto two different types of nanometre-sized clay: sepiolite and bentonite. The previously reported treatment to activate the filler surfaces was based on the possible interactions that occur between the co-catalyst (MAO) and the functional groups on the surface of these clays. The experimental method consisted of exposing dissolved MAO to a certain amount of nanoclay suspended in toluene as explained above. The used nanoclays presented high acidity due to their hydroxyl groups $(-\mathrm{OH})$, which act as Brønsted acids. Methyl-Aluminium ( $\mathrm{Me}-\mathrm{Al}$ ) bonds presented in MAO may react with these hydroxyl groups, thus anchoring the MAO by creating a covalent Aluminium-Oxygen ( $\mathrm{Al}-\mathrm{O}$ ) bond. This treatment is successful not only because of the amount of MAO is anchored but also because the supported MAO remains available (thanks to its high sensitivity to impurities) during the polymerization process to form active species in the presence of the metallocene catalyst. Therefore, a heterogeneous catalyst is obtained that counteracts the drawbacks of homogeneous catalysis. Unlike the Ziegler-Natta catalyst, the use of metallocene catalysts in in situ polymerization can be very sensitive to the filler surface. This sensitivity is due to the immobilization process included the co-catalyst instead of a single catalyst molecule as in most in situ polymerization processes $[35,36]$. Moreover, immobilizing the co-catalyst onto the clay was carried out without previous clay calcination. This is an advantage over conventional catalyst supports.

The active species for the polymerization process were formed when the surface-anchored active co-catalyst was exposed to the metallocene catalyst during the reaction. This procedure should encourage the polymer to grown directly from the filler surface. This process helped to efficiently exfoliate or defibrillate the nanoparticles, thus promoting clay de-agglomeration and dispersion, especially in the laminar case (BET) where an exfoliated structure was necessary.

First, neat polyethylene was synthesized in the absence of filler to evaluate the experimental conditions for the synthesis. For comparison, two nanocomposites with different types of clay but with similar experimental conditions were synthesized to study the influence of shape and morphology of these silicates on the molar mass and other properties $\left(S N 2 P E \_2: 1 \_50{ }^{\circ} \mathrm{C}\right.$ and BN2PE_2:1_50 ${ }^{\circ} \mathrm{C}$ sample with SEP and BET, respectively). Table 2 shows the results for all samples studied.

The results show an evident increase in the molar mass of both nanocomposites in comparison with unloaded polyethylene, which is consistent with the general results obtained from ethylene polymerization with the supported metallocene catalyst, where it was demonstrated that the clay was protecting the active species from other chemical species during the polymerization process. A decrease in chain transfer and termination reactions therefore occurred, which was produced by the polymer growth from the active metallocene centres located on the nanoclay surface [30,32]. This decrease gave an advantage to propagation reactions allowing the increase in molar mass.

The presence of sepiolite in the polymerization medium has a more noticeable effect on the increase in molar mass of the nanocomposites in comparison with the effect of the bentonite. This effect is probably due to the different shape and morphology of these clays. Bentonite has a considerably lower population of hydroxyl surface groups than those on sepiolite to function as polymerization active sites. Additionally, the $-\mathrm{OH}$ groups can be localized inside the layers of the bentonite, where they are unable to create new active sites. Consequently, fewer active sites were formed on this type of clay (see Table 1) and thus, lower productivity was obtained, which led to a highly loaded nanocomposite (see Table 2).

However, not only does the bentonite have fewer active sites to synthesize new polymeric chains but also this laminar nanoclay has less surface area and a lower aspect ratio compared with the fibrillar type sepiolite. The sepiolite's needlelike morphology better enables the monomer to not only interact with the external surface of the clay but also to penetrate into its structure, which achieves better dispersion into the synthesized polymeric matrix. Additionally, sepiolite is well known to have a higher swelling capacity than bentonite in the presence of organic solvents such as toluene or hexane. This higher swelling capacity enables greater dispersion of sepiolite because solvent molecules can better penetrate the swelled clay during the polymerization, which thus encourages the nascent polymer chains to defibrillate the clay. In addition, the swellability enables more adsorption of the catalyst onto the clay structure because the catalyst is also dissolved in the polymerization medium. This allows the increase in active sites on the sepiolite tunnels. In conclusion, both the high aspect ratio and the swellability are responsible for the better dispersion and distribution of the fibrillar clay in the nanocomposites, as demonstrated in the TEM microphotographs shown in Fig. 1(a) and (b). Although the bentonite particles were well distributed in the polymerized matrix, their layers did not exhibit exfoliation. This demonstrates that the shape of clay had a high influence in the nanocomposites obtained by in situ polymerization.

Finally, note that organically modified clays can swell when exposed to organic solvent. The level of dispersion can be correlated to the solubility parameters of the solvent and organically modified organoclay. Therefore, treating the bentonite with tertiary or quaternary ammonium salts can improve its swellability in the polymerization medium [37,38]. However, a prior modification is necessary for this type of clay. 
Table 2

Thermal, mechanical and morphological properties of the as-obtained nanocomposites under different polymerization conditions.

\begin{tabular}{|c|c|c|c|c|c|c|c|c|c|c|c|c|}
\hline & Sample $^{\mathrm{b}}$ & $\begin{array}{l}T_{m} \\
\left({ }^{\circ} \mathrm{C}\right)\end{array}$ & $\begin{array}{l}T_{C} \\
\left({ }^{\circ} \mathrm{C}\right)\end{array}$ & $\begin{array}{l}\Delta H_{m} \\
(\mathrm{~J} / \mathrm{g})\end{array}$ & $\begin{array}{l}X \\
(\%) \\
\end{array}$ & $\%$ Clay $^{\mathrm{C}}$ & Productivity $^{\mathrm{d}}$ & $\begin{array}{l}\text { Mw } \\
(\mathrm{g} / \mathrm{mol})\end{array}$ & P.I. & $\begin{array}{l}\text { Modulus } \\
(\mathrm{MPa})\end{array}$ & $\begin{array}{l}\sigma_{y} \\
(\mathrm{MPa})\end{array}$ & $\% \varepsilon_{b}$ \\
\hline Type of clay & $\begin{array}{l}\text { PE_50 }{ }^{\circ} \mathrm{C} \\
\text { SN2PE_2:1_50 }{ }^{\circ} \mathrm{C} \\
\text { BN2PE_2:1_50 }{ }^{\circ} \mathrm{C}\end{array}$ & $\begin{array}{l}140.3 \\
143.1 \\
138.4\end{array}$ & $\begin{array}{l}106.0 \\
106.1 \\
110.6\end{array}$ & $\begin{array}{l}234 \\
233 \\
180\end{array}$ & $\begin{array}{l}85.6 \\
85.8 \\
62.4\end{array}$ & $\begin{array}{r}- \\
- \\
5.4 \pm 0.4 \\
6.2 \pm 0.1\end{array}$ & $\begin{array}{l}3.23 \times 10^{3} \\
2.77 \times 10^{3} \\
2.01 \times 10^{3}\end{array}$ & $\begin{array}{l}2.4 \times 10^{5} \\
4.3 \times 10^{5} \\
3.0 \times 10^{5}\end{array}$ & $\begin{array}{l}2.63 \\
2.10 \\
4.86\end{array}$ & $\begin{array}{l}- \\
- \\
-\end{array}$ & $\begin{array}{l}- \\
- \\
-\end{array}$ & $\begin{array}{l}- \\
- \\
-\end{array}$ \\
\hline $\begin{array}{l}\text { Temperature } \\
\text { profile }^{\mathrm{e}}\end{array}$ & $\begin{array}{l}\text { PE_50-100 }{ }^{\circ} \mathrm{C} \\
\text { SN2PE_2:1_50-100 }{ }^{\circ} \mathrm{C} \\
\text { PE_100 }{ }^{\circ} \mathrm{C} \\
\text { SN2PE_2:1_100 }{ }^{\circ} \mathrm{C}\end{array}$ & $\begin{array}{l}139.8 \\
144.1 \\
132.4 \\
135.0\end{array}$ & $\begin{array}{l}108.0 \\
108.8 \\
103.7 \\
104.3\end{array}$ & $\begin{array}{l}284 \\
254 \\
260 \\
273\end{array}$ & $\begin{array}{l}96.9 \\
92.1 \\
88.5 \\
93.1\end{array}$ & $\begin{array}{l}- \\
- \\
- \\
- \\
2.9 \pm 0.7\end{array}$ & $\begin{array}{l}8.51 \times 10^{3} \\
9.12 \times 10^{3} \\
7.68 \times 10^{3} \\
1.95 \times 10^{4}\end{array}$ & $\begin{array}{l}9.6 \times 10^{4} \\
1.9 \times 10^{5} \\
4.4 \times 10^{4} \\
6.5 \times 10^{4}\end{array}$ & $\begin{array}{l}4.14 \\
3.20 \\
3.90 \\
2.84\end{array}$ & $\begin{array}{l}- \\
- \\
-\end{array}$ & $\begin{array}{l}- \\
- \\
-\end{array}$ & $\begin{array}{l}- \\
- \\
- \\
-\end{array}$ \\
\hline $\begin{array}{l}\text { Additional co- } \\
\text { catalyst }\end{array}$ & $\begin{array}{l}\text { SN2PE_2:1_50-100 }{ }^{\circ} \mathrm{C} \\
\text { NMSN2PE_2:1_50-100 }{ }^{\circ} \mathrm{C}\end{array}$ & $\begin{array}{l}144.1 \\
139.9\end{array}$ & $\begin{array}{l}108.8 \\
109.0\end{array}$ & $\begin{array}{l}254 \\
212\end{array}$ & $\begin{array}{l}92.1 \\
80.5\end{array}$ & $\begin{array}{l}3.5 \pm 0.7 \\
7.5 \pm 0.8\end{array}$ & $\begin{array}{l}9.12 \times 10^{3} \\
6.68 \times 10^{3}\end{array}$ & $\begin{array}{l}1.9 \times 10^{5} \\
7.1 \times 10^{5}\end{array}$ & $\begin{array}{l}3.20 \\
2.03\end{array}$ & $\begin{array}{l}1351 \pm 40 \\
1276 \pm 21\end{array}$ & $\begin{array}{l}31 \pm 2 \\
35 \pm 1\end{array}$ & $\begin{array}{l}508 \pm 19 \\
383 \pm 10\end{array}$ \\
\hline $\begin{array}{l}\text { Sepiolite } \\
\text { treatment }^{\mathrm{f}}\end{array}$ & $\begin{array}{l}\text { PE } \\
\text { N2PE_1:2 } \\
\text { N2PE_1:1 } \\
\text { N2PE_2:1 } \\
\text { N2PE_4:1 }\end{array}$ & $\begin{array}{l}139.8 \\
142.2 \\
142.2 \\
144.1 \\
144.7\end{array}$ & $\begin{array}{l}108.0 \\
107.9 \\
107.2 \\
108.8 \\
105.4\end{array}$ & $\begin{array}{l}284 \\
266 \\
261 \\
254 \\
216\end{array}$ & $\begin{array}{l}96.9 \\
94.4 \\
93.1 \\
92.1 \\
80.6\end{array}$ & $\begin{array}{l}- \\
1.8 \pm 0.2 \\
2.3 \pm 0.4 \\
3.5 \pm 0.7 \\
8.4 \pm 0.1\end{array}$ & $\begin{array}{l}8.50 \times 10^{3} \\
1.10 \times 10^{4} \\
9.30 \times 10^{3} \\
9.12 \times 10^{3} \\
4.80 \times 10^{3}\end{array}$ & $\begin{array}{l}9.6 \times 10^{4} \\
- \\
- \\
1.9 \times 10^{5} \\
3.7 \times 10^{5}\end{array}$ & $\begin{array}{l}4.14 \\
- \\
- \\
3.20 \\
2.06\end{array}$ & $\begin{array}{l}- \\
- \\
- \\
1351 \pm 40 \\
1143 \pm 38\end{array}$ & $\begin{array}{l}- \\
- \\
- \\
31 \pm 2 \\
27 \pm 1\end{array}$ & $\begin{array}{l}- \\
- \\
- \\
508 \pm 19 \\
168 \pm 21\end{array}$ \\
\hline $\begin{array}{l}\text { Same quantity of } \\
\text { final load }\end{array}$ & $\begin{array}{l}\text { N4PE_2:1 } \\
\text { N2PE_4:1 }\end{array}$ & $\begin{array}{l}144.7 \\
144.7\end{array}$ & $\begin{array}{l}108.7 \\
105.4\end{array}$ & $\begin{array}{l}230 \\
216\end{array}$ & $\begin{array}{l}90.5 \\
80.6\end{array}$ & $\begin{array}{l}8.3 \pm 0.2 \\
8.4 \pm 0.1\end{array}$ & $\begin{array}{l}6.59 \times 10^{3} \\
4.80 \times 10^{3}\end{array}$ & $\begin{array}{l}2.8 \times 10^{5} \\
3.7 \times 10^{5}\end{array}$ & $\begin{array}{l}2.76 \\
2.06\end{array}$ & $\begin{array}{l}1560 \pm 23 \\
1143 \pm 38\end{array}$ & $\begin{array}{l}30 \pm 2 \\
27 \pm 1\end{array}$ & $\begin{array}{l}310 \pm 12 \\
168 \pm 21\end{array}$ \\
\hline $\begin{array}{l}\text { Amount of final } \\
\operatorname{load}^{f}\end{array}$ & $\begin{array}{l}\text { N0.5PE_2:1 } \\
\text { N1PE_2:1 } \\
\text { N2PE_2:1 } \\
\text { N3PE_2:1 } \\
\text { N4PE_2:1 } \\
\text { N5PE_2:1 }\end{array}$ & $\begin{array}{l}144.4 \\
144.9 \\
144.1 \\
144.6 \\
144.7 \\
142.1\end{array}$ & $\begin{array}{l}109.1 \\
109.6 \\
108.8 \\
108.9 \\
108.7 \\
109.8\end{array}$ & $\begin{array}{l}290 \\
286 \\
254 \\
241 \\
230 \\
194\end{array}$ & $\begin{array}{l}99.6 \\
98.3 \\
92.1 \\
91.3 \\
90.5 \\
80.5\end{array}$ & $\begin{array}{r}1.0 \pm 0.3 \\
1.6 \pm 0.1 \\
3.5 \pm 0.7 \\
4.5 \pm 0.1 \\
8.3 \pm 0.2 \\
17.6 \pm 0.3\end{array}$ & $\begin{array}{l}1.12 \times 10^{4} \\
1.36 \times 10^{4} \\
9.12 \times 10^{3} \\
7.06 \times 10^{3} \\
6.59 \times 10^{3} \\
3.22 \times 10^{3}\end{array}$ & $\begin{array}{l}- \\
- \\
1.9 \times 10^{5} \\
- \\
2.8 \times 10^{5} \\
5.1 \times 10^{5}\end{array}$ & $\begin{array}{l}- \\
- \\
3.20 \\
- \\
2.76 \\
2.23\end{array}$ & $\begin{array}{l}1116 \pm 16 \\
1200 \pm 49 \\
1351 \pm 40 \\
1395 \pm 32 \\
1560 \pm 23 \\
1820 \pm 50\end{array}$ & $\begin{array}{l}29 \pm 1 \\
31 \pm 1 \\
31 \pm 2 \\
29 \pm 2 \\
30 \pm 2 \\
32 \pm 2\end{array}$ & $\begin{array}{l}307 \pm 24 \\
374 \pm 18 \\
508 \pm 19 \\
410 \pm 29 \\
310 \pm 12 \\
105 \pm 22\end{array}$ \\
\hline
\end{tabular}

${ }^{\text {a }}$ Polymerization conditions: ethylene pressure: 3 bar, $\mathrm{V}: 1 \mathrm{~L}$ (toluene as solvent), polymerization time: 15 min, additional MAO:catalyst ratio was [Al]/ [Zr]:1000 (Zr: $\left.7 \times 10^{-6} \mathrm{~mol}\right)$ and reaction temperature: $50^{\circ} \mathrm{C}$ in isothermal conditions.

b Nomenclature: PE: polyethylene matrix, S: sepiolite or B: bentonite, N2: nanocomposite obtained with $2 \mathrm{~g}$ of initial clay, $2: 1$ corresponding to the ratio [g initial clay:mL MAO solution] used in the prior treatment to the clay, and NM is the nanocomposite without additional MAO.

c Calculated by TGA at $900{ }^{\circ} \mathrm{C}$.

d $\mathrm{kg}_{\mathrm{PE}} /\left(\mathrm{mol}_{\mathrm{Zr}} \cdot\right.$ bar.h)

e PE_50-100 ${ }^{\circ} \mathrm{C}$ and SN2PE_2:1_50-100 ${ }^{\circ} \mathrm{C}$ sample were synthesized under non-isothermal profile under the same condition described in a part.

${ }^{\mathrm{f}}$ All samples were obtained under non-isothermal polymerization and with additional MAO.
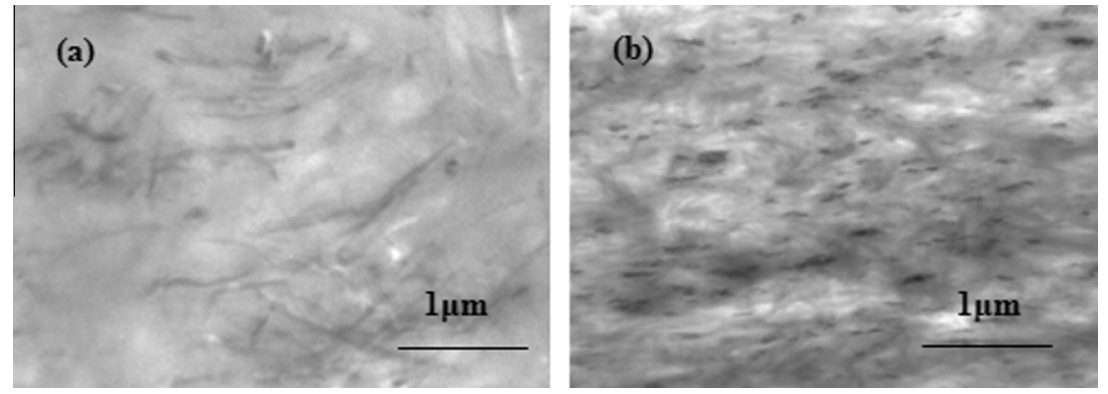

Fig. 1. TEM microphotographs of (a) SN2PE_2:1 and (b) BN2PE_2:1 samples.

The results of the final properties of nanocomposites, including the productivity and final loading associated with amount of active sites, are in agreement with literature (i.e., more active sites present in the polymerization process increase the activity of the catalyst and the amount of synthesized as well as decrease the filler concentration in the final nanocomposite) [39]. Surprisingly, the relationship between the number of active sites and the molar mass are not consistent because higher molar mass and a narrower distribution are obtained in the nanocomposite that has the clay with the highest amount of grafted co-catalyst $\left(S N 2 P E_{-} 2: 1 \_50^{\circ} \mathrm{C}\right.$ ). This result demonstrated that the type of support used as the filler (in terms of shape and morphology) acted as an important influence on the catalytic activity of the metallocene system.

Additionally, the clay shape seemed to have a significant effect on the thermal properties of the nanocomposites. The bentonite was a greater impediment to the crystallization of the polymer chains than the sepiolite was because the first one has less surface area. This is why this nanocomposite showed a significant decrease in enthalpy, crystallinity and melt temperature compared with those of neat PE (see Table 2). 
Exfoliating the layers of laminar clay particles (BET) during the polymerization reaction must be taken into account to achieve a good dispersion in the synthesized matrix. Furthermore, this dispersion should be achieved not only in the polymerization reactor but also in the subsequent melting and moulding processes. Fig. 2 shows a significant increase in the interlayer distance even though the bentonite has few active sites for polymerization. This result may be interpreted as a good BET distribution in the matrix. Note that this structure was maintained even after the material was extruded, although no exfoliated bentonite was observed. Additionally, the dispersion of sepiolite into the nanocomposite cannot be observed in an X-ray pattern due to its fibrillar structure, although perfectly separated fibres can be observed in the TEM microphotographs.

\subsection{Non-isothermal polymerization}

One of the most important results thus far is attributed to the increase in molar mass when the nanocomposites were obtained with sepiolite. The increase in the molar mass and the narrowing of the mass distribution in these materials are interesting from the point view of the mechanical properties that were attained. However, these materials may exhibit poor processability. Taking these considerations into account, a non-isothermal process was designed to give higher activation energy of the catalysts during the reaction and thus broaden the molar mass distribution while avoiding the use of hydrogen or co-monomers as transfer agents.

Temperature is known to be an experimental parameter that influences the activation of the catalyst and the productivity of the polymerization reaction. Temperature therefore has a marked effect on the structure of the final synthesized polymer [40]. To select the temperature of our in situ polymerization process that offers the greatest benefit in the control of the molecular weight two factors must be considered. The first consideration is that at low temperature, the catalyst works as a homogeneous single site system. The second one is that working at high polymerization temperatures significantly increases the activity of the catalyst, which makes it unstable. Moreover, at high temperatures, the reaction involves an unexpectedly more complex process than in homogeneous catalysis, as several active sites for polymerization may coexist [41]. The temperature increase leads to a sharp decrease in the molar mass of synthesized polymers. When they are polymerized with certain metallocene catalysts between $80-100{ }^{\circ} \mathrm{C}$, liquid oligomers with $2000 \mathrm{~g} \mathrm{~mol}^{-1}$ of Mw are obtained [42].

This new method was designed to obtain nanocomposites with a wide distribution of molar mass, which allowed a balance between the mechanical and rheological properties of the synthesized material, thanks to the benefits of a combination of high- and low-molecular-weight chains [43,44]. Synthesizing polymers with bi-modal and broad molecular weight distributions has been studied since the publications of the first patents in the early 1990s [45-47]. This research has evolved from mixing catalysts to designing continuous processes to synthesize differently sized polymer chains in two stages. Adapting these methodologies to an in situ polymerization process is a key strategy to improve the nanoclay's effect on the molecular weight of the polyethylene matrix.

Fig. 3(a) shows the GPC curves for two similar nanocomposites synthesized isothermally at $50{ }^{\circ} \mathrm{C}\left(\mathrm{N} 2 \mathrm{PE2}: 1 \_50{ }^{\circ} \mathrm{C}\right)$ and $100{ }^{\circ} \mathrm{C}\left(\mathrm{N} 2 \mathrm{PE} 2: 1 \_100^{\circ} \mathrm{C}\right)$ and a nanocomposite polymerization non-isothermally (N2PE2:1_50-100 $\left.{ }^{\circ} \mathrm{C}\right)$. These graphs confirmed that the isothermal polymerization (at $50^{\circ} \mathrm{C}$ ) produced the highest molar mass and a narrower mass distribution.

Viscosity in the molten state of the nanocomposite $N 2 P E 2: 1 \_50^{\circ} \mathrm{C}$ was measured at $2.18 \mathrm{~kg} / 190{ }^{\circ} \mathrm{C}\left(\mathrm{I}_{2}\right)$ and at $10 \mathrm{~kg} / 190{ }^{\circ} \mathrm{C}$ $\left(\mathrm{I}_{10}\right)$, obtaining a fluidity of $0.5 \mathrm{~g} 10 \mathrm{~min}^{-1}$ and $7 \mathrm{~g} 10 \mathrm{~min}^{-1}$, respectively. These values indicated that these materials had a melt flow rate (MFR) between 14 and 16, which corresponds to the typical behaviour of ultra-high molecular weight polyethylene. As expected, the uniform distribution of the molar mass in this sample (Mw/Mn $\sim 2$ ) involved a highly ordered polymerization mechanism and a single active site, and thus this nanocomposite is not interesting from the point of view of their processability.

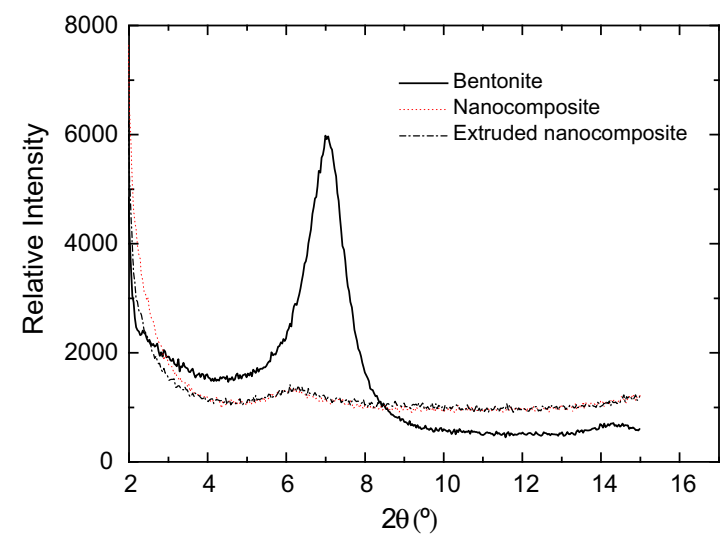

Fig. 2. XRD patterns of BET before and after in situ polymerization. 
Temperature profile effect
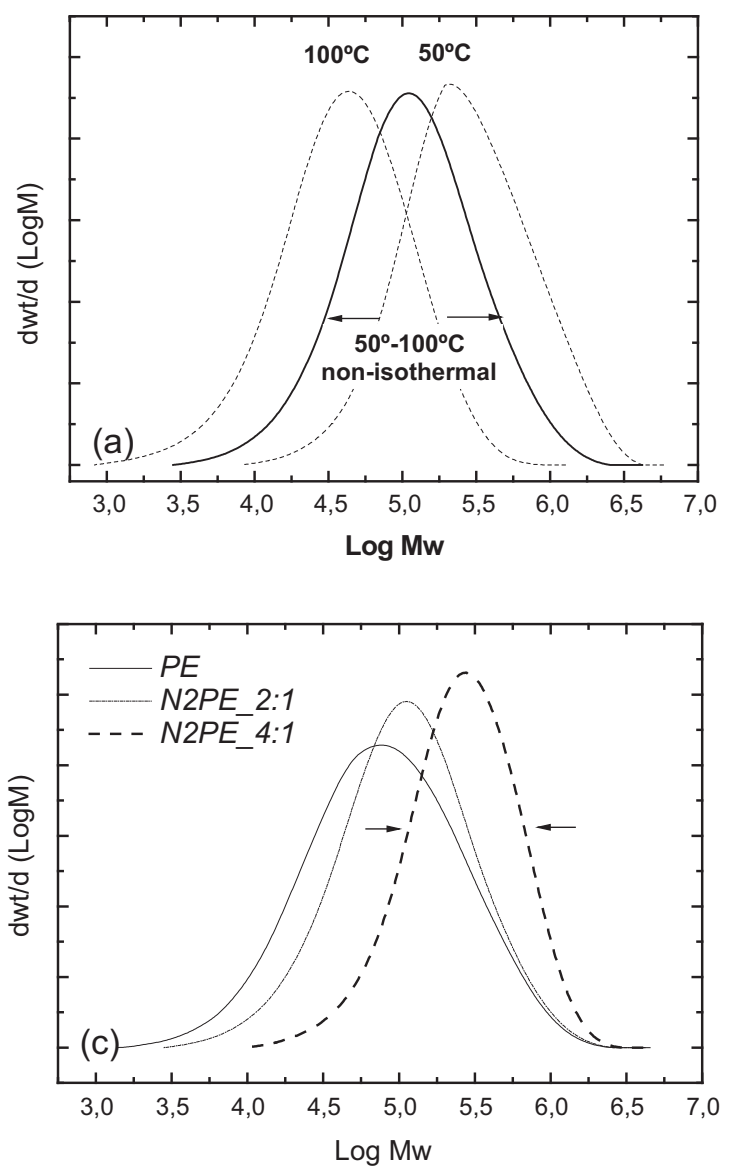

Sepiolite treatment effect
Additional co-catalyst effect
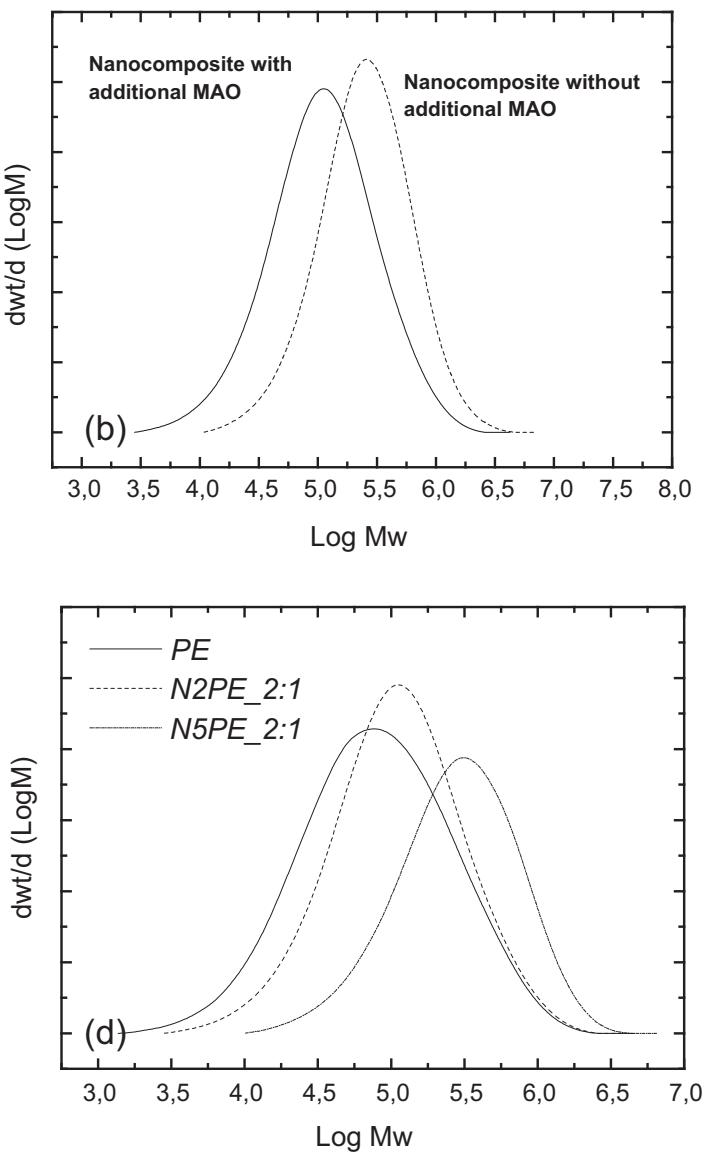

Amount of final load effect

Fig. 3. GPC curves. Effect of the (a) temperature profile, (b) additional co-catalyst, (c) sepiolite treatment, and (d) amount of final load on the molar mass and its distribution.

By increasing the temperature to $100{ }^{\circ} \mathrm{C}$ in an isothermal polymerization, the molar mass decreased by approximately an order of magnitude, i.e., short chains were polymerized. The melt flow index of this nanocomposite was $32 \mathrm{~g} 10 \mathrm{~min}^{-1}$, i.e., it flowed nearly 60 times faster than its analogous nanocomposite polymerized at $50{ }^{\circ} \mathrm{C}$. The melt elasticity and the mechanical properties were sacrificed by this large decrease in the molar mass, although the fluidity was improved. The properties of the synthesized compounds are presented in Table 2.

One of the most significant results is the changes in reaction productivity. The isothermal polymerization at $50{ }^{\circ} \mathrm{C}$ is very slow; after 20 min of reaction, a lower amount of synthesized polymer was obtained compared with that obtained by the non-isothermal process. This behaviour is reflected in the high percentage of sepiolite in the nanocomposite, with a final load of $5.4 \mathrm{wt} . \%$.

The isothermal polymerization at $100{ }^{\circ} \mathrm{C}$ is only able to produce small chains soluble in the reaction, which facilitates the contact of the catalysts with the monomer. Therefore, this condition increased the productivity and consequently, the final amount of clay in the nanocomposite was low (2.9 wt.\%). These small chains melt at low temperature, which increases their apparent mobility, and thus the nanoclay could act as a nucleant agent to increase their crystallinity (see $N 2 P E 2: 1 \_100{ }^{\circ} \mathrm{C}$ in Table 2).

Compared with neat PE, the most important feature of nanocomposites obtained by the non-isothermal profile is associated with the decrease in the enthalpy and the increases in the crystallinity and melt temperature. These differences may be due to the competition between the effects of the increase in molar mass because of the presence of clay and the reduction of the molar mass because of the higher reaction temperature. The balance of these effects provided a structure with fewer crystals (i.e., a decrease in enthalpy), but in turn these structures have a greater lamellar thickness (melting temperature increase). All of these effects were induced by the greater ability of sepiolite to nucleate low molecular weight chains. 


\subsection{Additional co-catalyst}

Thus far, the nanocomposites were synthesized with a small quantity of an additional co-catalyst not anchored onto the clay to broaden the molar mass distribution and increase the mobility of the chains. These features improve the fluidity during processing without sacrificing the melt elasticity. A nanocomposite without additional free MAO (NMSN2PE_2:1_50$100^{\circ} \mathrm{C}$ ) was also synthesized to assess the effect of the MAO on the molar mass.

Fig. 4 shows a diagram that explains the difference between polymerization from sepiolite with anchored MAO and from added free MAO. The polymer chains growing from the nanofiller replicate its form, and the polymerization is governed by uninterrupted propagation, which promotes the formation of these large chains. The chains that are formed from the added free MAO suffer the termination processes typical of homogeneous catalysis.

The total amount of catalyst used in all the reactions remained unchanged. Thus, some of the catalyst was activated by the additional free MAO and the remaining amount by the anchored MAO. Therefore, the $\mathrm{Al} / \mathrm{Zr}$ ratio that compares the amount of additional MAO with the amount of catalyst was very small (1000) compared with the ratio used in this polymerization type [40].

Fig. 3(b) shows the GPC curves of the two compounds synthesized with and without additional MAO. The decrease in the molar mass due to the addition of free MAO and the increase in the polydispersity index indicate that shorter chains were synthesized. However, part of the additional MAO could possibly react with the treated sepiolite present in the reaction and graft onto the clay before creating new sites for homogeneous polymerization. This grafting may be why the distribution of molar mass is not divided into two different populations when the polymerization combines heterogeneous and homogeneous systems.

Although the goal is to reduce the molar mass, the other properties that determine the end use of the nanocomposite must not be neglected. Therefore, we evaluated and compared the thermal and mechanical characteristics of the samples SN2PE_2:1_50-100 ${ }^{\circ} \mathrm{C}$ and NMSN2PE_2:1_50-100 ${ }^{\circ} \mathrm{C}$. The results are presented in Table 2.

One of the collateral consequences of adding additional MAO is the increase in the productivity because there are more active sites during the synthesis. This increase in productivity decreases the final amount of nanofiller in the nanocomposite. This is why the sample with additional MAO has a sepiolite content of $3.5 \mathrm{wt} . \%$ while the sample without additional MAO has a content $7.5 \mathrm{wt} . \%$. The additional MAO in the reaction decreases the concentration of the impurities that are the responsible for carrying out propagation reactions, which also contributes to decreasing the productivity in the sample NMSN2PE_2:1_50-100 ${ }^{\circ} \mathrm{C}$.

Another important difference between these two materials lies in their melt properties. The nanocomposite without the additional MAO loses the ability to crystallize due to lower mobility that yields larger chains in its structure, which thus lowers the melt temperature.

In terms of mechanical properties, the NMSN2PE_2:1_50-100 ${ }^{\circ} \mathrm{C}$ nanocomposite shows a strong interaction between the polyethylene matrix and nanofiller. Although a high percentage of deformation is not achieved, it tolerates higher stress before breaking compared with the SN2PE_2:1_50-100 ${ }^{\circ} \mathrm{C}$ sample. This behaviour is associated with the effective transfer of stress between the matrix and filler when a normal force is applied. Therefore, sepiolite offers a strong resistance to deformation, which causes a fracture due to the inability of this sample to continue deforming.

According to the rheological properties, the sample with additional MAO exhibits a melt flow index of $7.3 \mathrm{~g} 10 \mathrm{~min}^{-1}$ $\left(2.16 \mathrm{~kg} / 190^{\circ} \mathrm{C}\right)$, while the sample without additional MAO, as expected, does not flow because all its chains are linked to the sepiolite. The low fluidity exhibited in standard measurement conditions clearly limits the use of this material.

The narrower molar mass distribution and the lack of fluidity in this sample indicates that controlling the amount of additional MAO in the reaction is more important than the non-isothermal profile for control of molecular weight.

Finally, it is important to note that synthesizing PE chains directly from the clay surface without using additional MAO is a clear indication that anchoring the MAO onto sepiolite is effective because it attaches to the active co-catalyst for polymerization.

\subsection{Modification clay}

Four sepiolite nanocomposites treated with different amounts of MAO were polymerized to study the influence of the amount of co-catalyst in the polymerization medium. All samples were non-isothermally polymerized with additional MAO.

The results demonstrated that the MAO treatment applied to the clay mainly determines the amount of filler remaining in the final nanocomposite (see Table 2). The more MAO is anchored onto the clay, the greater the number of sites available to synthesize new polymeric chains. This increases the productivity of the system, and the amount of filler in the nanocomposite is further diluted. On the other hand, low amounts of MAO on the clay surface causes more deactivation of the catalyst by impurities and moisture present on the clay, which leads to a lower quantity of synthesized polymer matrix.

Additionally, there are small changes in the melt and crystallization temperatures of the nanocomposites compared with those of neat PE. Other authors have achieved a more pronounced nucleant effect in matrices obtained by in situ polymerization with other nanometre size clays $[29,48,49]$. In addition, the decrease in the amount of MAO on the clay not only attenuates the nucleant effect but also produces significant losses in the melt enthalpy and in the crystallinity of the nanocomposite. This unexpected behaviour is demonstrated by the nanocomposites with the lower amount of MAO on the sepiolite surface, which exhibited the greatest increase in molar mass (see Fig. 3(c)). 


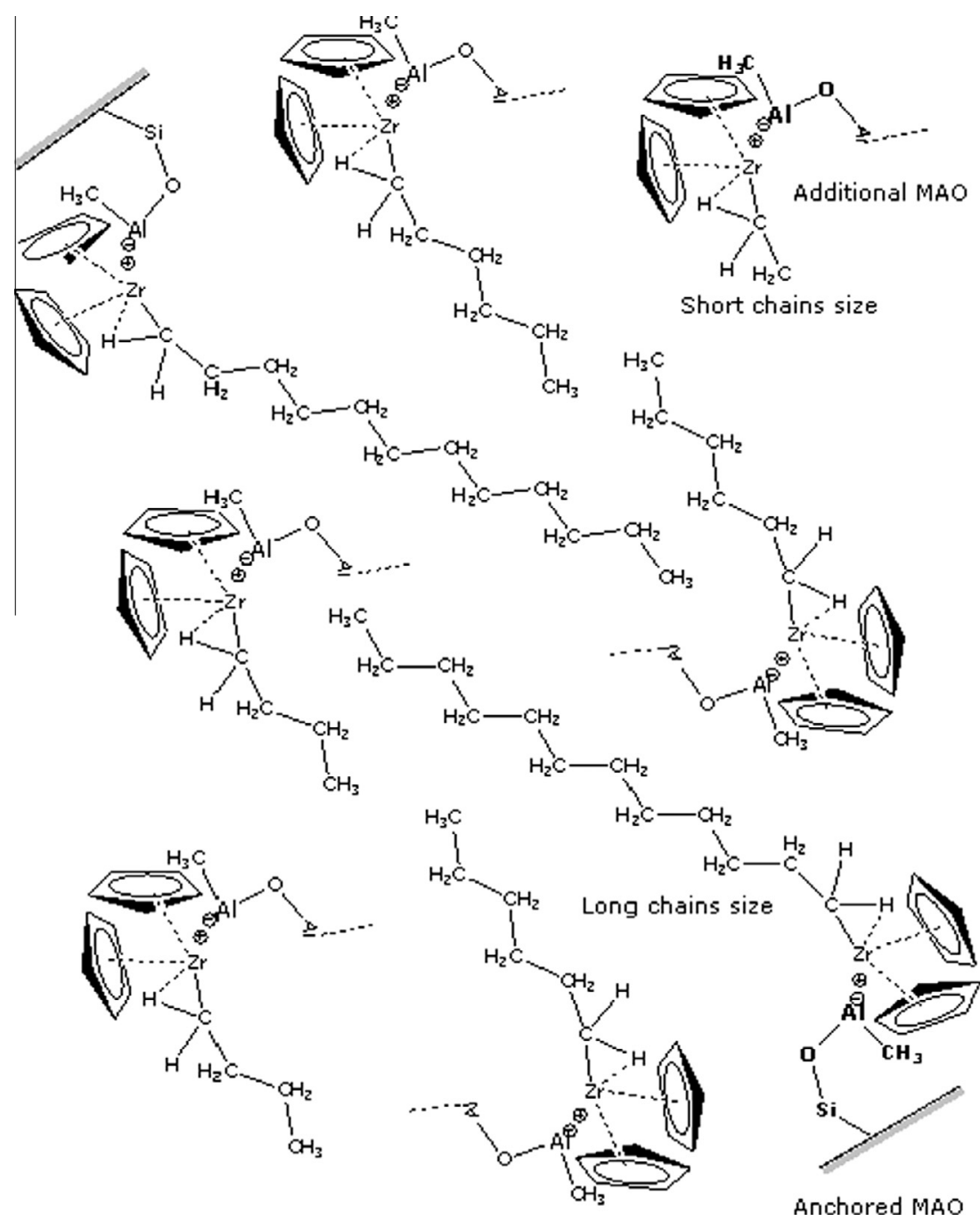

Fig. 4. Structure of the polymeric chain synthesized from both anchored and additional free MAO.

It is important to remember that the properties of the nanocomposites obtained by in situ polymerization depend on all of the experimental process parameters for the synthesis. However, the results reported in Fig. 5 show that properties are only dependent on the final amount of filler, without taking into consideration that clays on nanocomposites were activated in different proportions. The amount of MAO anchored to the clay surface will determine the amount of untreated clay, which will largely determine the mechanical properties of the nanocomposite.

To demonstrate that the final properties of the nanocomposites depend not only on the final amount of filler but also on the experimental parameters used in the synthesis (e.g., the amount of initial clay fed into the reactor, polymerization times, filler treatment, etc.), two materials (N2PE_4:1 and N4PE_2:1) were compared, each with the same amount of final filler $(\sim 8 \mathrm{wt} . \%)$ but with different treatments and amounts of clay fed into the reaction. This combination enabled obtaining the same final amount of sepiolite in the nanocomposites. The results of the characterization are shown in Table 2.

The Young's modulus $(E)$ and elongation at break $\left(\varepsilon_{b}\right)$ were higher in the N4PE_2:1 sample, which indicates the greater interaction between the matrix and the load. This nanocomposite is capable of transferring the force more efficiently because the clay treatment created more active sites, which decrease the molar mass without affecting the crystallinity of the nanocomposite. This reaction is also more productive (30\%), suggesting that more polymer chains are able to grow from this sepiolite. These results confirm that more active sites on the clay lead to an increase in the matrix-clay interaction.

Therefore, the treatment ratio used in the following study was clay:MAO =2:1. The selection of this treatment is based on balancing the obtained properties and the amount of MAO used, considering that using large amounts of MAO is not an economically preferable treatment. 


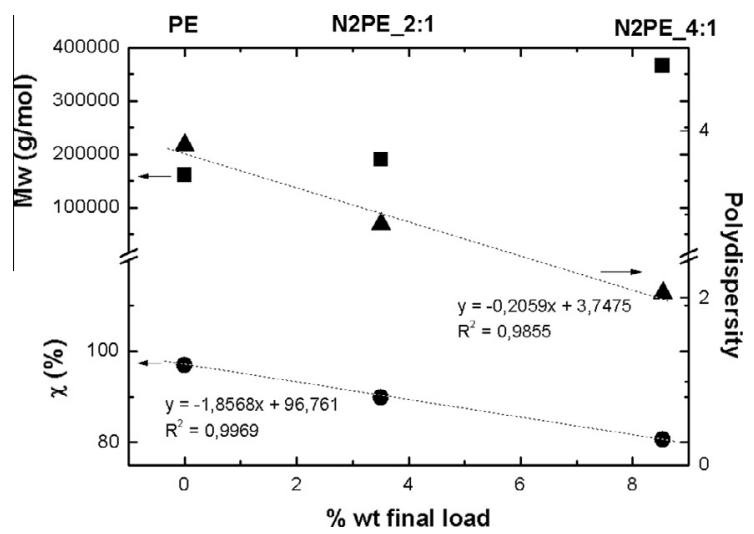

Fig. 5. Relationship between the morphological properties and the amount of final load in the synthesized nanocomposites.

\subsection{Influence of the final amount of clay in the nanocomposite}

Six materials with $0.5,1,2,3,4$, and $5 \mathrm{~g}$ of initial sepiolite were polymerized and characterized, and the results are presented in Table 2. Most importantly, only slight variations exist in the thermal properties of the synthesized nanocomposites. The difference between the melt temperatures of neat PE and its nanocomposites is less than $5{ }^{\circ} \mathrm{C}$, which is not observed in the crystallization temperatures. This variation is shown in the DSC curves in Fig. 6. It seems that the hierarchy imposed on the synthesis by the metallocene catalyst prevails over the effect of having filler in the synthesized matrix.

The SSA endotherms in Fig. 6(b) show that there is still a unique population of crystals, regardless of the final amount of clay in the nanocomposite. This shows that increasing the amount of sepiolite in the material does not promote new crystallite regions capable of increasing the melt temperature.

On the other hand, as shown in Table 2, low final percentages of sepiolite (1-1.5 wt.\%, which corresponds to N0.5PE and N1PE samples) have a nucleant effect, which slightly increases the crystallinity and melt enthalpy compared with those of neat PE. However, once the amount of final filler in the nanocomposite has reached a critical value, the nucleation effect is stopped because the filler behaves as a defect in the crystal system, and therefore the melt enthalpy begins to gradually decrease.

This behaviour is absolutely dependent on the type of catalyst employed; the one used in this study promotes a very high crystallinity, which makes generating a more crystalline system untenable because of the clay nucleant effect. An schematic illustration of the effects of polymerizing ethylene with sepiolite at various concentrations is shown in Table 3 .

None of these effects significantly changes the melt temperatures of the crystal sections, indicating that there are no major changes in the lamellar thickness. Although there are no major changes in the structure of crystals, the increase in the molar mass should be considered. The GPC curves in Fig. 3(d) show the increase in the molar mass with the amount of sepiolite in the nanocomposite, as mentioned in the previous section.

These new larger chains, which were created because the clay prevents termination reactions, are barred by their own size from incorporating into the crystal structure. This behaviour again explains the loss of crystallinity when the load increases. The effect on the reaction productivity because of the higher amount of MAO accompanying the higher amount of clay should also be considered (i.e., if the amount of clay increases, the total amount of MAO also increases proportionately). The more co-catalyst is present in the medium, which increases the amount of produced PE, the higher is the amount of clay, i.e., higher concentrations of $-\mathrm{OH}$ groups on the surface. This may increase the termination reactions in addition to anchoring MAO onto fewer accessible sites in the clay [30]. Therefore, there is a compromise between the effects on productivity when the amount of clay increases in the polymerization.

The phenomenon of higher productivity when a catalyst is supported is associated with the partial immobilization of catalyst onto the surface of the support that was treated with MAO [28]. The heterogeneous system stabilizes the catalyst in the reaction, and it is widely reported that polymerizations with supported catalysts need a $\mathrm{Al} / \mathrm{Zr}$ proportion considerably lower than in the homogeneous polymerizations [19]. However, in those papers the support was usually silica, which has a lower concentration of - $\mathrm{OH}$ groups and a considerably smaller surface area than sepiolite [50,51]. In addition, the amounts of substrate used as a support are between 10 and 20 times lower than the amounts used as a filler [52]. These differences work against the productivity of the polymerization of these nanocomposites.

The compromise that exists between the sums of effects (positive and negative) over the productivity using filler as supporting of catalyst system are reflected in Table 2. If the reactor is fed with low amounts of clay, there is an increase in the productivity compared with that of neat PE. On the other hand, greater amounts of sepiolite lead to a significant decrease in the amount of PE synthesized.

This phenomenon is also reflected in Fig. 7, which shows the relationship between the amount of clay initially fed into the reactor and the final filler amount in the nanocomposite. Low percentages of initial clay produce a proportional increase in 

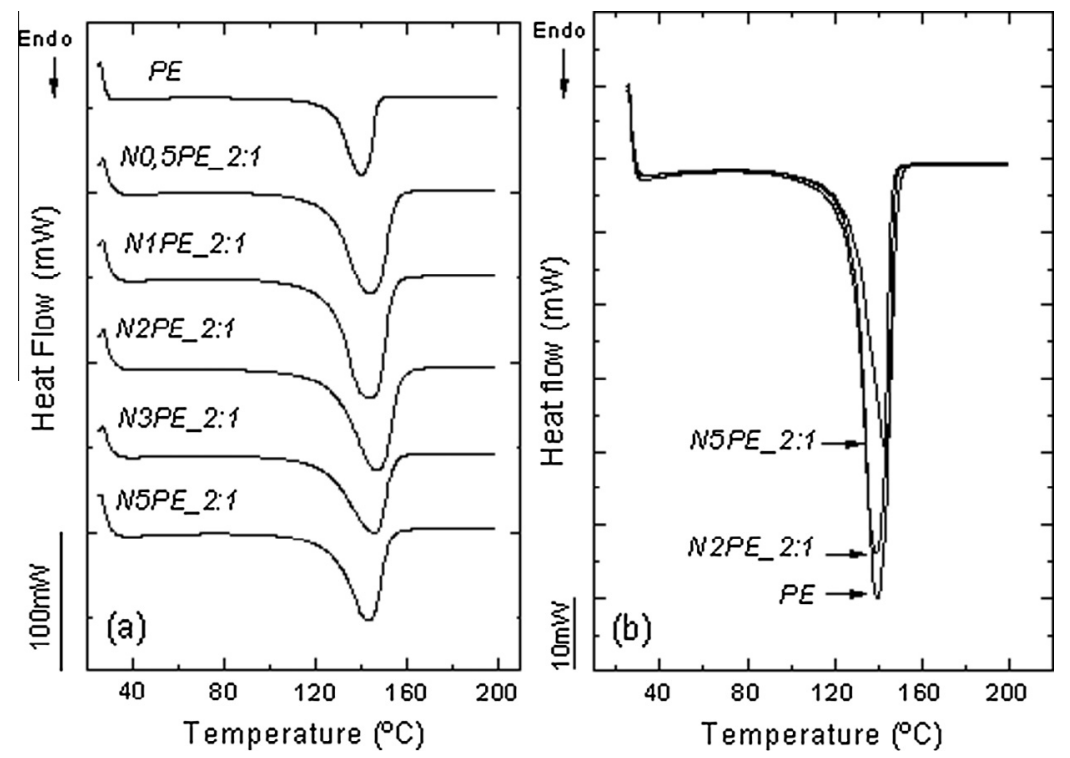

Fig. 6. (a) DSC curves and (b) overlapping SSA curves of PE nanocomposites obtained with various amounts of load.

the final clay material, but above a certain limiting load, the final percentage of sepiolite in the nanocomposite increases exponentially. This leads to a decrease in the quantity of PE synthesized. Saturating the reaction volume with the nanofiller increases the probability of contact between the growing chains and the hydroxyl groups, which leads to termination reactions, as discussed above.

Fig. 7 could be used to predict the amount of clay in the synthesized nanocomposite. Parameters such as the molar mass, mobility of chains and mechanical properties depend on the amount of filler in the nanocomposites. Remarkably, the main transition temperatures are frequently observed to be conditioned by the filler in nanocomposites prepared by melt processes. Differences in these thermal transitions can be expected in these materials because this method of synthesis involves a substantial increase in molecular weight, while in nanocomposites obtained by melt intercalation, the molecular weight is kept constant.

The storage modulus and $\tan \delta$ versus temperature are plotted in Fig. 8(a) and (b), respectively. These curves are useful to study the viscoelastic behaviour of the synthesized materials. A notable increase in the storage modulus is a common feature in nanocomposites with a small quantity of laminar silicates because these small amounts of load are enough to improve the mechanical properties of these materials [2]. The storage modulus calculated from the results of a dynamic test in Fig. 8(a) indicate that needle-like loads such as sepiolite may achieve the same effect as laminar silicates when they are used in an in situ polymerization process.

These graphs show that the PE homopolymer has a lower storage modulus than its nanocomposites. The nanocomposite with 4.5 wt.\% of clay (N2PE2:1) exhibits a more noticeable increment in this parameter than that of the sample with 17 wt.\% of this filler (N5PE2:1). However, despite the significant difference in final load in their structures, they do not exhibit a significant difference in the modulus.

The values of the storage modulus at different temperatures for each sample were presented in Table 4 . With respect to neat PE, the storage modulus at $-30{ }^{\circ} \mathrm{C}$ is over $40 \%$ higher for the nanocomposite with 3.5 wt.\% of clay (N2PE2:1) and almost $90 \%$ higher for the sample with 17 wt.\% of sepiolite (N5PE2:1). Considering the discussion above, the higher modulus of the material with $17 \mathrm{wt} . \%$ of load compared with the one loaded with $3.5 \mathrm{wt} . \%$ is not surprising. This difference is clear evidence that there is a compromise between the effects in synthesizing the nanocomposite with this method of synthesis; the loss of crystallinity counteracts the reinforcing effect of the filler on the matrix.

Note that the preliminary results showed that there is a filler load limit $(\sim 2 \mathrm{wt} . \%)$ and that above this limit, the crystallinity does not increase with the further addition of sepiolite. Therefore, the most effective improvement in the mechanical properties is achieved with low percentages of nanofiller, which leads to a more productive and economic procedure.

The dependence of $\tan \delta$ on temperature is shown in Fig. 8(b) to examine the main molecular transitions of the synthesized nanocomposites. The transitions in PE are typically found at $-110^{\circ} \mathrm{C},-20^{\circ} \mathrm{C}$ and $70{ }^{\circ} \mathrm{C}$ which are associated with $\alpha, \beta$, and $\gamma$ transitions, respectively [53].

Relaxation $\gamma$ is associated with the glass transition and therefore the movement of the amorphous fractions of the polymer matrix. Fig. 8(b) shows a significant difference in the temperature at which this transition occurs in the nanocomposite N5PE2:1 in comparison to neat PE, which demonstrates how the load restricts the mobility of the amorphous sections and the dissipation of energy due to the movements of these chains. Moreover, this is reflected in a change in the modulus, as mentioned above. 
Table 3

Representative schemes on the evolution of the polymer structure due to an increase in the load in the polymerization medium.

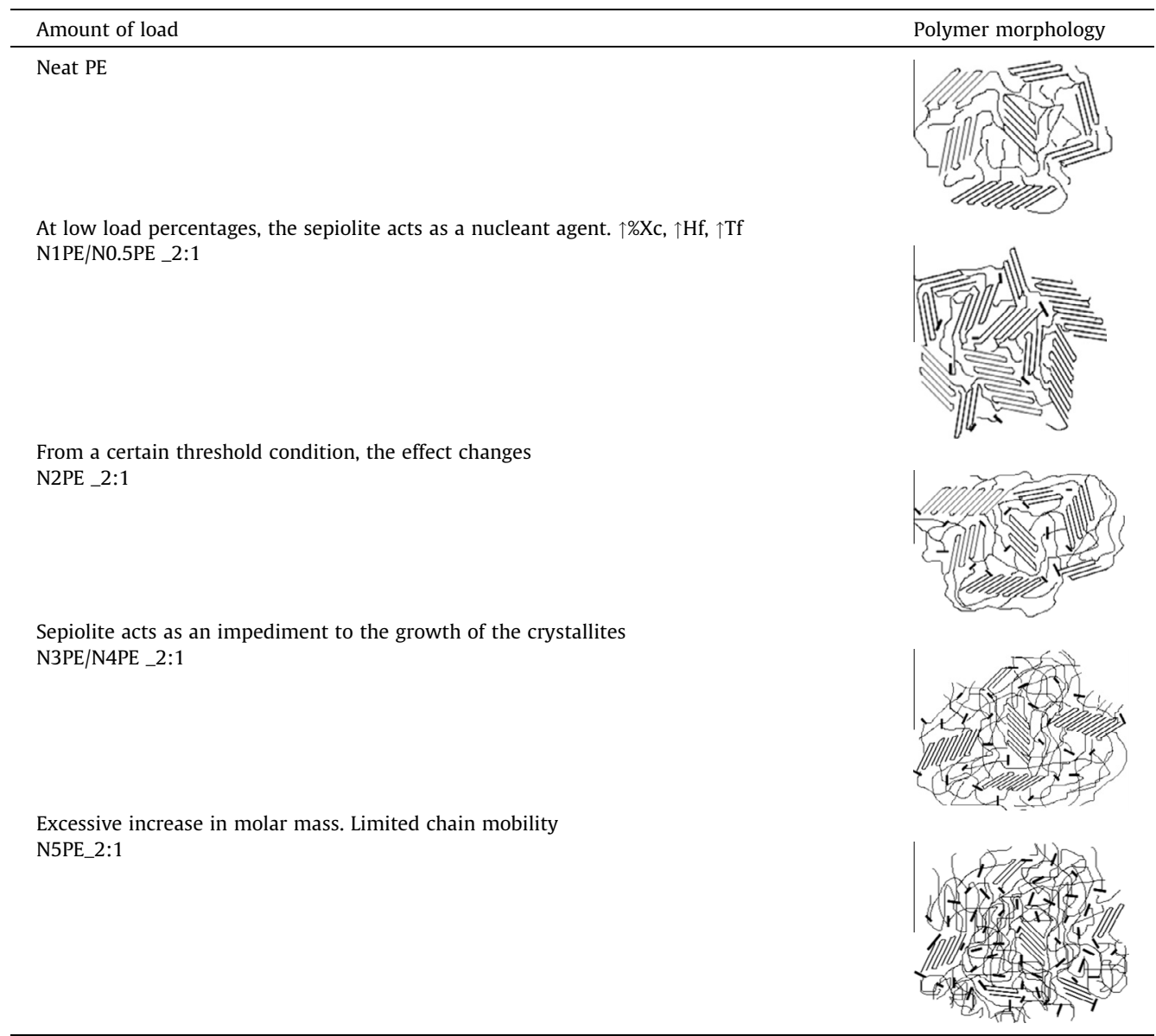

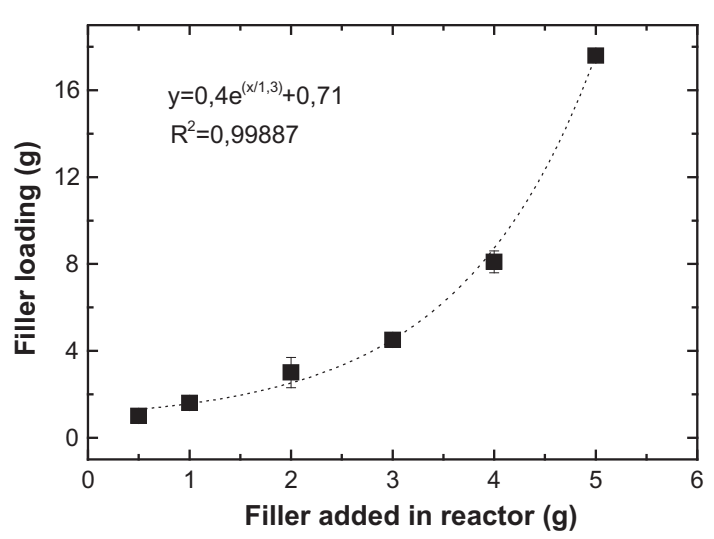

Fig. 7. Relationship between the amounts of clay fed into the reactor and the amount of final load present in the nanocomposite.

Remarkably, the nanocomposite N5PE2:1 possesses the highest proportion of amorphous zones (see Table 2) but at the same time the greatest restriction of mobility due to the high amount of clay. These results once again demonstrate the dual role played by sepiolite in in situ polymerization and this clay's significant effect on the molar mass of the final nanocomposites. 

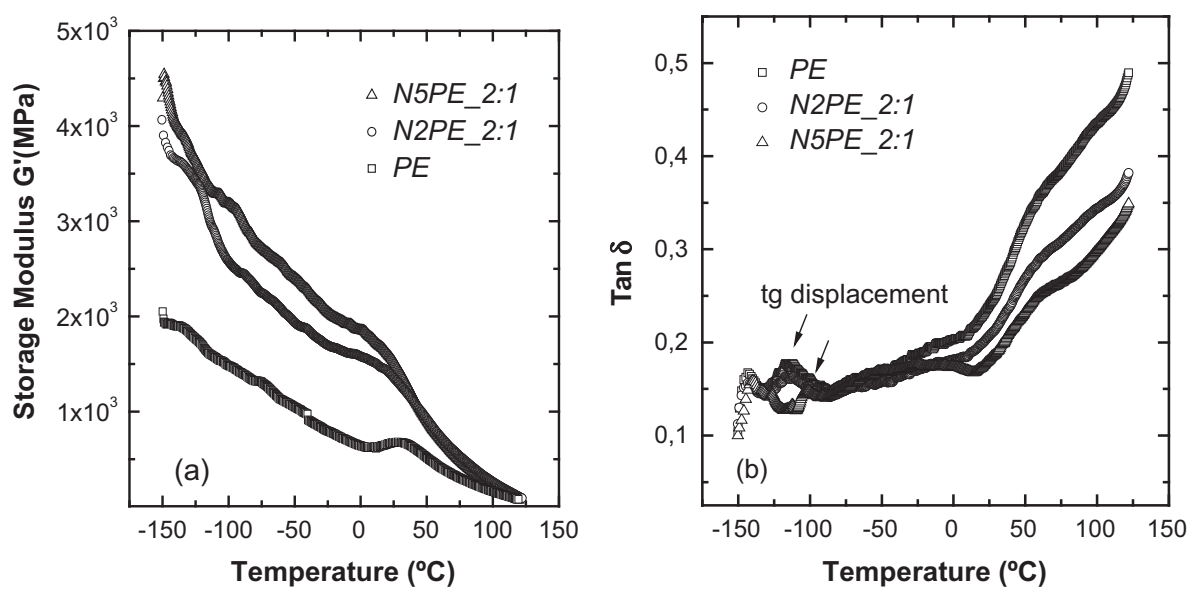

Fig. 8. Temperature dependence of (a) storage modulus and (b) $\tan \delta$ for PE and its nanocomposites.

Table 4

Dynamic storage modules of PE and its nanocomposites at various temperatures.

\begin{tabular}{llll}
\hline Sample & \multicolumn{2}{l}{ Storage module (GPa) } & $30^{\circ} \mathrm{C}$ \\
\cline { 2 - 4 } Name & $-80{ }^{\circ} \mathrm{C}$ & $-30{ }^{\circ} \mathrm{C}$ & 1.04 \\
\hline PE & 1.83 & 1.23 & 1.28 \\
N2PE_2:1 & 2.25 & 1.74 & 1.40 \\
N5PE_2:1 & 2.80 & 2.33 & \\
\hline
\end{tabular}

The next molecular transition appears at approximately $-20^{\circ} \mathrm{C}$. This transition is associated with the mobility limitations in the non-crystalline segments of the chains. This molecular relaxation, which is more typical of branched polyethylenes, is almost imperceptible in these materials.

Finally, the $\alpha$ transition is associated with intercrystalline relaxation and sliding chains. The intensity of this transition decreases in the N5PE2:1 sample because of the lower crystallinity compared with that of neat PE. Finally, the results show that both amorphous and crystalline areas were affected by the presence of clay. These results are also manifested in macroscopic mechanical tests. Table 2 reports the values of the main parameters measured in a uniaxial tensile test. The change in the Young's modulus shown in Table 2 is a widely reported phenomenon in nanocomposites obtained by various methods, different matrices, and with a wide variety of nanofillers [2]. However, when PE is synthesized with a very specific metallocene catalyst, as in this study, large increments in the modulus are not expected with the addition of in situ fillers because the matrix is highly crystalline, has a high molecular weight and high rigidity. Alexandre et al. reached this conclusion when the authors synthesized metallocene nanocomposites with a variety of nanosilicates (hectorite, kaolin and montmorillonite) with approximately $4 \mathrm{wt} . \%$ of final filler [23]. The properties of these materials did not exceed those of the neat polymer because it was obtained via metallocene synthesis.

The nanocomposites with low percentages of filler (4 wt.\%) obtained in this work exhibited a notable improvement in the modulus mainly due to our process control over the molecular weights of the matrix that was synthesized. Fig. 9 shows that the increment of the specific modulus is very pronounced at low percentages of incorporated sepiolite, and it exhibits a sigmoidal trajectory until it reaches a plateau where higher amounts of nanofiller offer no further improvements.

The pronounced increase in modulus at low filler percentages is due to the nucleant effect of the filler. Nevertheless, the further increments in the modulus at higher percentages of load is due to the resistance against deformation offered by the clay and due to the good interaction between the matrix and the clay to transmit the applied stress. This implies that only 3.5 wt.\% of filler (N2PE2:1) is necessary to achieve an increment of 30\% in the Young's modulus with respect to neat PE, while nearly 5 times more clay (N5PE2:1) is necessary for an increase of 60\%. In addition, these high percentages of clay lead to a detriment of other tensile properties such as tenacity.

On this last point, the percentage of elongation at break and the final tensile strength of these materials were also studied. Nanofillers are well known to improve the rigidity at the expense of reducing the elongation at break. Therefore, a progressive loss of ductility in the nanocomposites synthesized with increasing amounts of clay would be expected. However, as shown in Fig. 10, there is clearly a interplay between effects that make this relationship more complex.

At low percentages of clay, a significant decrease in the elongation at break is demonstrated (e.g., a loss of approximately $40 \%$ in the material NO.5PE was achieved). This is the unmistakable result of the nucleant effect that occurs when the polymerization takes place at low percentages of clay, as reported in the thermal parameters. From this point, the elongation at 


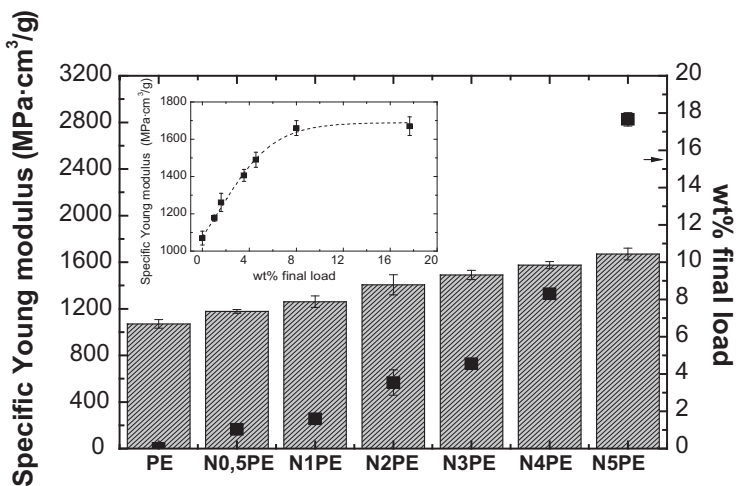

Fig. 9. Dependence of the specific Young module within the final load amount of the nanocomposites.

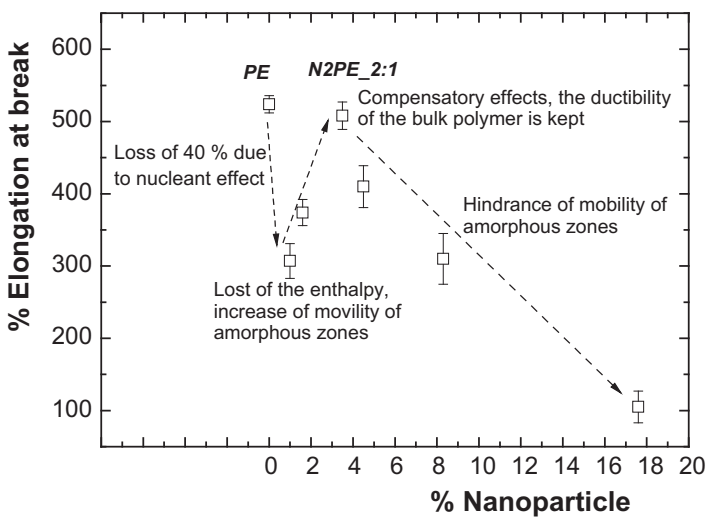

Fig. 10. Dependency of the \% elongation at break on the final load amount of the nanocomposites.

break begins to increase with increasing amounts of clay until it reaches a maximum. This increase is a result of the decrease in the enthalpy, which is associated with an increase in the amorphous sections and therefore with the mobility of the chains. When the amount of filler is above $3.5 \mathrm{wt}$ \% in nanocomposites (N2PE2:1), the mobility of the amorphous sections is conditioned, which results in a progressive loss of the elongation at break.

Table 3 shows the clear influence of the amount of sepiolite on the structure and on the properties of the synthesized nanocomposites. Therefore, it can be concluded that under these conditions, there is an optimal percentage of clay below which there is a significant increase in crystallinity and rigidity and hence a substantial loss of ductility. Above the optimal percentage of clay, the increment in the modulus is less pronounced because of the effect of clay on the change in molecular weight. At the same time, sections with low crystallinity form, and therefore, the mobility of the amorphous section are impeded by the saturation of filler, which leads to less tenacious materials.

Therefore, the optimum material is the nanocomposite synthesized with $2 \mathrm{~g}$ of initial sepiolite which achieved a $3.5 \mathrm{wt} . \%$ of nanofiller in the final structure. In this material, a constant elongation at break and an increase of $40 \%$ in the Young's modulus compared with that of neat PE is achieved. This surprising result is associated with a high dispersion of filler and good interaction between the matrix and sepiolite.

Evidence of good interaction between the sepiolite and PE is shown in Fig. 11. The nanocomposite stiffens in response to the normal force, which increases the maximum force applied to the material before breaking. This phenomenon is known as deformation hardening and is the result of the chains rearranging in the direction of the force applied during the test. This is evidence of how effectively the stress is transferred by the matrix to the filler, which allows the material to deform before breaking. The filler in the majority of nanocomposites concentrates force, which promotes the formation and propagation of cracks in the material in the early stages of tensile testing and thus leads to the loss in deformability of the materials.

Finally, the increase in the yield stress (see Table 2) confirms that applying a larger force is necessary to overcome the elastic transition of nanocomposites compared with the force necessary to deform PE, which once again demonstrates the good distribution and interaction of sepiolite with the synthesized matrix. This evidence is also confirmed by the TEM microphotographs shown in Fig. 1(a).

Regarding the microscopic tests, the N2PE_2:1 powder was observed by scanning electron microscopy (SEM) (see Fig. 12). Note that the powder has a needle-like structure. Based on the theory of the "replication of morphologies" that has been 


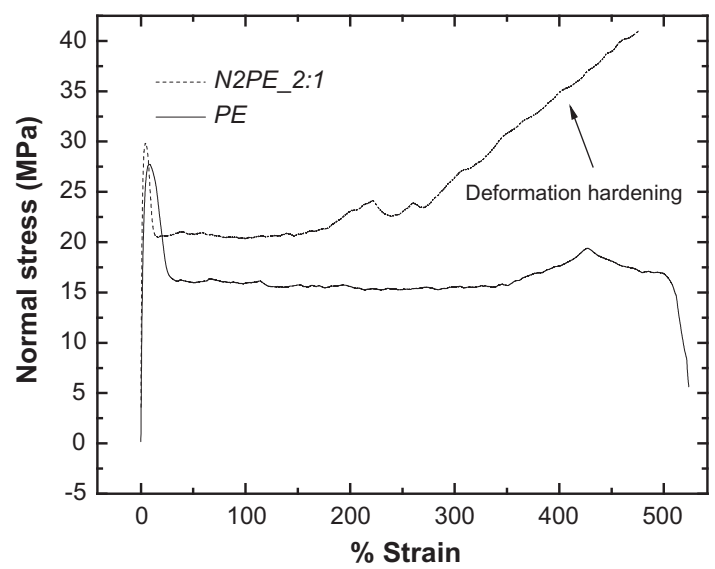

Fig. 11. Stress-strain curves from the tensile tests of neat PE and the N2PE_2:1 nanocomposite.

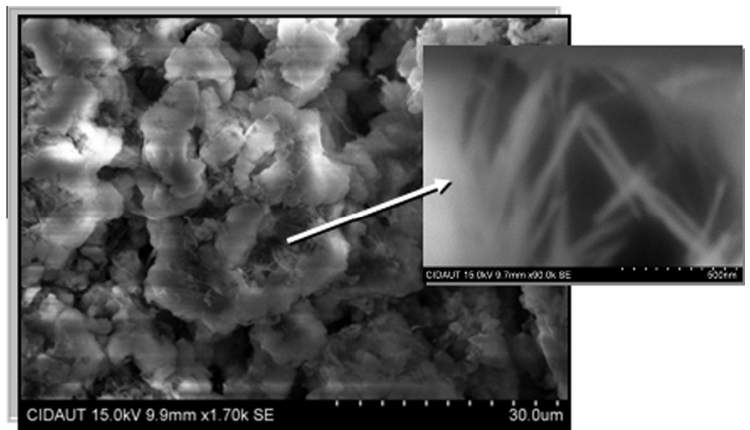

Fig. 12. SEM micrograph of the polymerization powders for sample N2PE.

Table 5

Results of the Soxhlet extraction of PE content in nanocomposites.

\begin{tabular}{ll}
\hline Sample & Insoluble fraction \\
\hline PE & 72 \\
N2PE_2:1 & 87 \\
N5PE_2:1 & 90 \\
\hline
\end{tabular}

observed in polyethylene synthesized with supported catalysts on silica [25,32], the needle-like structure obtained in this work could be due to a replication of the fibrillar structure of sepiolite. The lengths of these needles are on the same order of nanometres and there are clumps of several hundred nanometres, which could be the result of encapsulation suffered by the sepiolite fibres due to the growth of polymer chains into its structure.

Finally, a more quantitative way to assess the interaction between the synthesized matrix and sepiolite is to measure the proportion of insoluble PE in the nanocomposite via a Soxhlet extraction test in decalin [54]. Considering that sepiolite is an insoluble mineral, the polymer chains that are "linked" or that have a strong interaction with the clay will lose the ability to dissolve, and the weight percentage of the extracted sample will be low. The results of the extraction of neat PE and the N2PE and N5PE samples are compared in Table 5. The presence of clay leads to a more insoluble compound, not only due to the existing interactions but also due to the significant increase in molecular weight that was obtained by the addition of filler to the polymerization medium.

\section{Conclusion}

The results of this study have shown that the change in the molar mass in nanocomposites obtained by in situ polymerization depends on the type of clay used in the reaction medium. The needle-like shaped clays have a greater ability to 
anchor the MAO onto their structure than the laminar clays. This result is due to the former's greater surface area and higher number of -OH groups, which promotes the anchoring reaction. Although fibrillar clays have a higher number of active sites for polymerization, their morphology promotes a significant increase in the length of the chains that grow over them. It was demonstrated that the nascent chains prefer the propagation reactions and the replication of the elongated shape of the filler. Therefore, a non-isothermal polymerization method was designed with additional MAO to enable the control of the molecular weight. The results showed that the additional MAO increases the amount of short chains, which improves the rheological properties of the nanocomposites. On the other hand, it was shown that the increase in molar mass is inversely proportional to the number of active sites on the clay and directly proportional to the amount of initial filler added to the polymerization reactor.

Finally, it is important to highlight that fibrillar clays significantly change not only the molar mass of the nanocomposites obtained but also its structure. The sepiolite may have a nucleant effect when it is used in low proportions. Otherwise, the clay is a defect in the crystal system when it is used in higher quantities because of the increase in the amorphous regions. A balance between both effects facilitates a material with high rigidity and high tenacity. These results potentially transform the in situ polymerization approach into a way to obtain nanocomposites with excellent clay dispersion and a good balance between mechanical and rheological properties.

\section{Acknowledgement}

The authors acknowledge the financial support of "Ministerio de Economía y Competitividad - Spain" through the project MAT2011-28212.C02.

\section{References}

[1] Q. Wang, Z. Zhou, L. Song, H. Xu, L. Wang, J. Polym. Sci. Part. A: Polym. Chem. 42 (2004) 38-43.

[2] M. Alexandre, Mater. Sci. Eng. 28 (2000) 1-63.

[3] L. Qiu, W. Chen, B. Qu, Polymer 47 (2006) 922-930.

[4] L. Song, Y. Hu, S. Wang, Z. Chen, W.J. Fan, Mater. Chem. 12 (2002) 3152-3155.

[5] E. Yildirid, N. Miskolczi, J.A. Onwudili, K.E. Nemeth, P.T. Williams, J. Soja, Composites Part B 78 (2015) $393-400$.

[6] K. Wang, I. Chung, M. Jang, J. Keum, H. Song, Macromolecules 43 (2002) 5529-5535.

[7] Y. Lee, K. Wang, C. Park, M. Sain, J. Appl. Polym. Sci. 103 (2007) 2129-2134.

[8] R. Truss, T. Yeow, J. Appl. Polym. Sci. 100 (2006) 3044-3049.

[9] A. Guimont, E. Beyou, P. Alcouffe, P. Cassagnau, A. Serghei, G. Martin, P. Sonntag, Polymer 55 (2014) $22-28$.

[10] Y. Chen, Y. Qi, Z. Tai, X. Yan, F. Zhu, Q. Xue, Eur. Polym. J. 48 (2012) 1026-1033.

[11] D. Liu, R.T. Olsson, M.S. Hedenqvist, U.W. Gedde, Eur. Polym. J. 66 (2015) 67-77.

[12] A. He, L. Wang, J. Li, J. Dong, C. Han, Polymer 47 (2006) 1767-1771.

[13] L.M. Wei, T. Tang, B.T. Huang, J. Polym. Sci., Part A: Polym. Chem. 42 (2006) 941-949.

[14] F. Shehzad, S.T. Thomas, M.A. Al-Harthi, Thermochim. Acta (2014) 226-234.

[15] A. Berlin, S. Volfson, N. Enikolopian, S. Negmatov, Principles of Polymer Composites, vol. 2, Springer-Verlag, Berlin, 1996, pp. 125-145. Chapter 3.

[16] J. Rong, Z. Jing, X. Hong, W. Zhang, China Petrochemical Corporation (Beijing, CN) Patent US 6444742, 2002.

[17] D. García-López, J.F. Fernández, J.C. Merino, J. Santarén, J.M. Pastor, Compos. Part B-Eng. 45 (2011) $459-465$.

[18] T. McKenna, J. Soares, Chem. Eng. Sci. 56 (2001) 3931-3949.

[19] G. Hlatky, Chem. Rev. 100 (2000) 1347-1376.

[20] Z. Xuejing, S. Madri, J. Chadwick, J. Loos, Macromolecules 38 (2005) 4673-4678.

[21] A. Michael, G. Beyer, C. Henrist, R. Cloots, A. Rulmont, R. Jérôme, P. Dubois, Macromol. Rapid. Commun. 22 (2001) 643-646.

[22] A. Michael, M. Pluta, P. Dubois, R. Jérôme, Macromol. Chem. Phys. 202 (2001) 2239-2246.

[23] M. Alexandre, T. Sun, J. Garces, R. Jérôme, P. Dubois, Polymer 43 (2002) 2123-2132.

[24] A. Michael, P. Dubois, R. Jerome, M. Garcia-Marti, T. Sun, J. Garces, D. Millar, A. Kuperman, United States The Dow Chemical Company, Patent U.S. 6465543,2002

[25] W. Kaminsky, A. Funck, K. Wiemann, Macromol. Symp. 239 (2006) 1-6.

[26] W. Kaminsky, A. Funck, Macromol. Symp. 260 (2008) 1-8.

[27] B. Paredes, J. Soares, Macromol. Symp. 257 (2007) 103-111.

[28] T. Halbach, R. Mülhaupt, Polymer 49 (2008) 867-876.

[29] S. Nikkhah, S. Ramazani, H. Baniasadi, F. Tavakolzadeh, Mater. Des. 30 (2009) 2309-2315.

[30] P. Zapata, R. Quijada, C. Covarrubias, E. Moncada, J. Retuert, J. Appl. Polym. Sci. 113 (2009) $2368-2377$.

[31] T. Halbach, Y. Thomann, R. Mülhaupt, J. Polym. Sci., Part A: Polym. Chem. 46 (2008) 2755-2765.

[32] W. Li, A. Adams, J. Wang, B. Blümich, Y. Yang, Polymer 51 (2010) 4686-4697.

[33] K. Núñez, R. Gallego, J.C. Merino, J.M. Pastor, Appl. Clay Sci. 101 (2014) 73-81.

[34] K. Núñez, R. Gallego, J.C. Merino, J.M. Pastor, C. Alonso, Fundación Cidaut, Spain W02013-167764A1, 2012.

[35] M.R. Ribeiro, A. Deffieux, M.F. Portela, Ind. Eng. Chem. Res. 36 (1997) 1224-1237.

[36] L. Simplicio, F. Costa, J. Boaventura, E. Sales, S.J. Brandão, Mol. Catal. A-Chem. 216 (2004) 45-50.

[37] A. Maneshi, J. Soares, L. Simon, Macromol. Chem. Phys. 212 (2011) 216-228.

[38] Y. Choi, S. Young, A. Shin, J. Soares, Macromol. Chem. Phys. 211 (2010) 1026-1034.

[39] W.J. Kaminsky, Polym. Sci. Pol. Chem. 42 (2004) 3911-3921.

[40] Y. Kissin, Alkene polymerization reactions with transition metal catalysts, Studies in surface science and catalysis, USA, 2008.

[41] H. Frauenrath, H. Keul, H. Höcker, Macromol. Chem. Phys. 202 (2001) 3551-3559.

[42] Z. Xia, G. Odian, A. Rossi, J. Polym. Sci., Part A: Polym. Chem. 38 (2000) 3802-3811.

[43] K. Nileshkumar, L. Balzano, W. Gerrit, R. Sanjay, J. Chadwick, Macromol. React. Eng. 3 (2009) $448-454$.

[44] F. Alt, L. Bohm, H. Enderle, J. Berthold, Macromol. Symp. 163 (2001) 135-144.

[45] Phillips Petroleum Company, Patent U.S. 4939217, 1990.

[46] Mobil Oil Corporation, Patent U.S. 5032562, 1991.

[47] Union Carbide Chemicals and Plastics Technology Corporation, Patent U.S. 5070055, 1991.

[48] M. Trujillo, M.L. Arnal, A.J. Muller, E. Laredo, S. Bredeau, D. Bonduel, P. Dubois, Macromolecules 40 (2007) 6268-6276. 
[49] G. Leone, F. Bertini, M. Canetti, L. Boggioni, P. Stagnaro, I.J. Tritto, Polym. Sci. Pol. Chem. 46 (2008) 5390-5403.

[50] S. Collins, K. Mark, D. Holden, Macromolecules 25 (1992) 1780-1785.

[51] R. Van Grieken, A. Carrero, I. Suarez, B. Paredes, Eur. Polym. J. 43 (2007) 1267-1277.

[52] C. Ekrachan, J. Bunjerd, P. Piyasan, Chem. Eng. Sci. 62 (2007) 899-905.

[53] H. Ying-Juan, Q. Ya-Wei, D. Jin-Yong, Z. Xutao, H. Xuteng, J. Appl. Polym. Sci. 123 (2012) 3106-3116.

[54] A. Shin, L. Simon, J. Soares, G. Scholz, Polymer 44 (2003) 5317-5321. 